\title{
Pôles de compétitivité : les systèmes productifs recomposés par les réseaux d'innovation
}

Competitiveness clusters: innovation networks and production system

\section{Antoine Grandclément}

\section{(2) OpenEdition}

\section{Journals}

Electronic version

URL: http://journals.openedition.org/rge/5128

DOI: $10.4000 /$ rge. 5128

ISSN: 2108-6478

Publisher

Association des géographes de l'Est

\section{Printed version}

Date of publication: 15 October 2014

ISSN: 0035-3213

Electronic reference

Antoine Grandclément, «Pôles de compétitivité : les systèmes productifs recomposés par les réseaux d'innovation », Revue Géographique de l'Est [Online], vol. 54 / 1-2 | 2014, Online since 15 October 2014, connection on 10 December 2020. URL : http://journals.openedition.org/rge/5128 ; DOI : https:// doi.org/10.4000/rge.5128

This text was automatically generated on 10 December 2020.

Tous droits réservés 


\title{
Pôles de compétitivité : les systèmes productifs recomposés par les réseaux d'innovation
}

\author{
Competitiveness clusters: innovation networks and production system
}

\author{
Antoine Grandclément
}

\section{Introduction}

1 Lancée en 2005, la politique des pôles de compétitivité a souvent été appréhendée par les inflexions qu'elle introduit dans les politiques industrielles et d'aménagement du territoire, conduisant par exemple Élie Cohen à parler de «nouvelle politique industrielle " (Cohen, 2007). La place centrale donnée à l'innovation et la volonté initiale de concentrer les moyens sur un nombre réduit de pôles, pour la plupart métropolitains et concentrés dans le champ des hautes technologies (Blanc, 2004), ont pourtant été remis en question par la labellisation de 71 pôles dans des secteurs et des territoires extrêmement divers (Albertini, 2006). La définition élaborée par la Datar (Délégation interministérielle à l'aménagement et l'attractivité régionale) a donné lieu à des lectures tout aussi diverses, certains voyant dans les pôles une «forme hybride d'agglomération des activités à mi-chemin entre le district industriel et le cluster " (Boquet et al. 2009, p228), tandis que d'autres soulignaient l'originalité de ces pôles qui ne sont à proprement parler «ni des clusters scientifiques à l'allemande (réunissant des chercheurs du public et du privé), ni des clusters industriels à l'italienne (reposant sur des liens inter-entreprises), ni des écosystèmes correspondant à des logiques d'agglomération des ressources comme dans les technopôles " (Fen-Chong et Weil 2008, p.4).

2 Définis comme « la combinaison, sur un espace géographique donné, d'entreprises, de centres de formation et d'unités de recherche publiques ou privées, engagés dans une démarche partenariale destinée à engager des synergies autour de projets communs au caractère innovant » (Cahier des charges de l'appel à projet), les pôles semblent relever 
du classique triptyque recherche/industrie/formation et du modèle de la proximité spatiale. Ils s'éloignent pourtant du modèle des clusters (Guillaume, 2008) par la volonté de l'État de promouvoir un changement d'échelle et par la place faite à la mise en réseau des acteurs industriels et scientifiques au sein de projets de recherche collaborative (Plunket, Torre, 2009). La volonté de ne pas opposer de barrière de nature géographique à la mise en réseau des acteurs s'est notamment incarnée dans la formule énigmatique de « territoires-réseaux » proposée par la Datar, un temps rebaptisée Diact (Délégation interministérielle à l'aménagement et la compétitivité des territoires), pour qualifier les pôles (Grandclement, 2012). Elle met en tension une logique territoriale d'ancrage des entreprises et l'insertion dans des réseaux à plus petite échelle, toutes deux supposées contribuer à faire émerger des "masses critiques" compétitives à l'échelle internationale. Le terme même de pôle est sans doute davantage l'expression d'une forme de marketing territorial qu'une indication réelle sur l'organisation spatiale des acteurs ou d'éventuelles logiques de polarisation.

3 Au-delà de ces débats sur la nature de cette politique nationale se pose la question de sa mise en œuvre plus grande échelle. Largement fondée lors de ces différentes phases sur le modèle de l'appel à projet, elle a effet laissée une grande latitude aux acteurs locaux et régionaux dans la construction des futurs pôles, notamment dans la définition de leur périmètre industriel et géographique. Au flou relatif du cahier des charges rédigé par l'État s'ajoute la grande diversité des lectures que les acteurs régionaux en ont faites, qu'il s'agisse des acteurs privés ou des collectivités territoriales. Une tension apparaît donc entre la dimension prescriptive de la politique nationale et du modèle qu'elle suggère et sa mise en œuvre concrète.

4 Cet article se propose d'interroger cette ambiguïté en analysant la mise en œuvre et le fonctionnement des pôles de compétitivité dans la région Provence-Alpes-Côte d'Azur (PACA). L'objectif est notamment de montrer que les pôles de compétitivité sont des acteurs importants de l'organisation des activités productives. Par une approche comparative, il s'agit également d'analyser comment les spécificités de chacun des pôles de compétitivité sont le fruit des mutations récentes des systèmes productifs régionaux. On s'appuie pour ce faire sur des entretiens réalisés auprès d'acteurs impliqués dans les pôles et sur une série d'observations non participantes entre 2009 et 2012 (correspondants CNRS des pôles, audit des pôles pour la Région PACA, construction de la Stratégie Régionale d'innovation).

5 La première partie présente les mutations spatiales et industrielles récentes des systèmes productifs de la région PACA qui constituent une clé de compréhension essentielle des modalités de mise en œuvre de la politique des pôles dans la région, présentée dans la deuxième partie. La troisième partie montre qu'au-delà des réseaux d'innovation auxquels ils donnent naissance, les pôles de compétitivité sont au cœur de la géographie de la production industrielle à l'échelle régionale.

\section{Les mutations des systèmes productifs régionaux en PACA}

6 Les pôles de compétitivité se sont construits sur les bases offertes par les industries régionales mais ils ont également été marqués par les formes d'organisation qui leur préexistaient. En ce sens, la filiation ne se limite pas à un paysage industriel donné à un instant $t$ mais intègre l'empreinte des évolutions anciennes et récentes des systèmes 
productifs qui ont dessiné ce contexte (Pecqueur, 2008). Ce terme de système productif révèle d'ailleurs à la fois les mutations de ce qu'on appelait de manière classique "l'industrie", autant que les renouvellements conceptuels qui tentent d'en rendre compte. Sylvie Daviet évoque ainsi l'image du chaos « pour un monde industriel chargé de transformations si profondes qu'on n'est plus certain de la pertinence du nom qu'on lui attribue et qu'on a du mal à s'accorder sur des substituts " (Daviet 2005b). La porosité des frontières entre les services et l'industrie, l'externalisation de nombreuses fonctions par les grandes entreprises et la réorganisation de leurs modes de production ont remis en question les catégories traditionnelles et imposé une redéfinition de l'industrie. Le concept de système productif se veut plus englobant et entend rendre compte des liens d'interdépendance entre des sphères auparavant séparées, la sphère de la production concrète, la sphère de la production abstraite (notamment les fonctions de conception) et enfin la sphère périproductive qui rassemble l'ensemble des services aux entreprises (Carroué, 2014). L'importance croissante de l'innovation est concomitante à ces transformations et n'y est certainement pas étrangère. La politique des pôles constitue d'ailleurs une reconnaissance du rôle désormais fondamental des fonctions de production abstraite.

\section{A. De la crise du système portuaire aux hautes technologies}

7 La géographie industrielle de la région Provence-Alpes-Côte d'Azur est marquée par deux phénomènes majeurs, l'opposition géographique entre l'est et l'ouest du territoire régional d'une part, les mutations profondes des systèmes productifs depuis un demisiècle d'autre part (Daumalin, Girard et Raveux 2003 ; Garnier 2011). Ces dernières ont en partie réactivé la structuration ancienne du territoire régional par les deux métropoles marseillaise et niçoise mais elles ont également affecté la géographie productive à échelle fine.

8 Au début de la seconde moitié du XXe siècle, le paysage industriel en PACA se caractérise à la fois par la faiblesse générale de l'industrie régionale et par le poids de pôles industriels dominés par des industries anciennes, profondément ancrées dans le territoire, mais qui connaissent des difficultés croissantes. Le complexe industrialoportuaire marseillais et les industries qui lui sont liées (Garnier et Zimmermann 2006) sont l'exemple le plus spectaculaire de cette situation. A contrario, l'industrie est peu développée sur le reste du territoire et les industries de pointe (Fache, 2002) pèsent peu à l'échelle régionale. La crise des industries anciennes a ainsi engendré de profondes mutations sectorielles, organisationnelles et spatiales qui ont renouvelé les systèmes productifs régionaux. L'ampleur des recompositions n'a toutefois pas été la même selon les secteurs et les territoires, conduisant à distinguer des « transitions-rupture » et des " transitions-continuité » (Garnier et al. 2004). Ces spécificités pèsent aujourd'hui sur les modes d'organisation des acteurs et des filières.

9 Les transformations les plus profondes ont touché les industries portuaires et notamment la construction navale depuis Marseille jusqu'aux chantiers de l'ensemble du littoral régional (La Ciotat, la Seyne-sur-Mer), ainsi que l'exploitation minière du bassin de Gardanne ${ }^{1}$. Les restructurations plus récentes du complexe militaroindustriel de la marine nationale incarnées par la transformation de la DCN (Direction des constructions navales) en acteur privé relèvent des mêmes processus. La disparition de ces activités et l'ampleur des impacts sur les sociétés et les territoires 
locaux ont conduit à une forte implication de l'État dans l'accompagnement social de la crise et dans la reconversion des territoires. Le constat du retard régional dans les hautes technologies a orienté l'action publique vers le développement d'industries de pointe dans le cadre de politiques industrielles nationales ambitieuses. L'implantation d'une industrie microélectronique (l'entreprise Eurotechnique devenue SGS Thomson puis ST Microelectronics) dans le bassin de Gardanne à Rousset en 1980, dans le cadre du plan Composants, illustre cette intervention de l'État-entrepreneur (Daviet 2003 ; Rychen et Zimmermann 2001). La création du technopôle de Sophia-Antipolis dans les Alpes-Maritimes au début des années 1970 (Laffitte 2003) poursuit les mêmes objectifs mais sous des formes différentes en mettant l'accent sur le projet d'aménagement de ce territoire et sur la place des organismes de recherche (Grondeau 2007). Dans les deux cas, le développement de ces greffons s'est appuyé sur des initiatives exogènes et sur l'implantation de grandes entreprises multinationales (Texas-Instrument et IBM dans les années 1960, Philips à la fin des années 1990, pour Sophia).

La rupture avec les anciens systèmes productifs n'a toutefois pas été totale. À Marseille, un tissu industriel endogène s'est ainsi constitué dans le secteur de la mer autour de nouvelles entreprises telles que la Comex et des spin-off ${ }^{2}$ auxquels elles ont donné naissance (Bertin, Cybernetix). Une dynamique similaire de création d'un tissu local de petites et moyennes entreprises et d'un système dense de relations entre les acteurs locaux s'est produite dans les anciennes villes-usines portuaires, sans que les grandes entreprises y jouent un rôle aussi important (Garnier et Mercier 2008). Les liens avec les universités et centres de recherche qui se sont développées parallèlement renforcent ces dynamiques, à l'image du rôle joué par l'IFREMER (Institut français de recherche pour l'exploitation de la mer) dans le domaine maritime. Dans d'autres secteurs, les mutations ont été beaucoup moins marquées, s'inscrivant plutôt dans une certaine continuité (Garnier et al. 2004). Le tissu agro-industriel de la basse vallée du Rhône (ibid.) ou l'industrie des arômes et parfums de Grasse (Mendez et Bardet 2009) relèvent davantage de ce second type de trajectoire, moins heurtée mais qui constitue souvent un frein à l'introduction d'innovations et à l'adaptation aux nouvelles conditions économiques.

\section{B. Une réorganisation spatiale à échelle fine}

11 Au-delà de ces secteurs et de ces territoires les plus emblématiques, les mutations des systèmes productifs ont également conduit à des réorganisations spatiales à échelle fine. Le cas de la région métropolitaine marseillaise illustre parfaitement ce processus de « recomposition et de re-territorialisation de l'appareil productif» (Garnier 2005, p. 368). La crise des industries traditionnelles liées au port est ainsi à l'origine d'un départ des activités du centre, laissant la place aux friches que l'opération d'urbanisme Euromed contribue aujourd'hui à requalifier. Un desserrement de l'activité (Garnier et Zimmermann 2006) s'est opéré parallèlement dans les périphéries marseillaises donnant naissance à un tissu d'entreprises de haute technologie, sans qu'aucun projet d'ensemble n'en soit à l'origine ${ }^{3}$. Le pays d'Aix-en-Provence du fait de ses aménités a polarisé ce processus qui se distingue surtout par la diversité des secteurs représentés (Garnier et Mercier 2008). La création de technopôles à Château-Gombert ou sur le site de l'Arbois en périphérie d'Aix-en-Provence illustre les transformations de la géographie industrielle de l'ouest de la région et l'émergence d'une dynamique métropolitaine. Bernard Morel évoque ainsi cinq événements fondateurs dans la 
«naissance » de la métropole marseillaise, qui concernent tous les périphéries : la création de la zone industrialo-portuaire de Fos, le soutien à l'aéronautique installée à Marignane, la création du Centre d'Études Nucléaires de Cadarache (CEN), l'installation du Centre d'Études Techniques de l'Équipement (CETE) sur la zone d'Aix-Les Milles et la création de la zone commerciale de Plan de Campagne ${ }^{4}$ (figure 1). Les temporalités disjointes de ces évolutions et l'émergence d'un appareil productif en apparence très segmenté convergent en fait vers une trajectoire métropolitaine (Morel 1999) que renforce la mise en réseau des acteurs.

Figure 1 : Les mutations du système productif marseillais

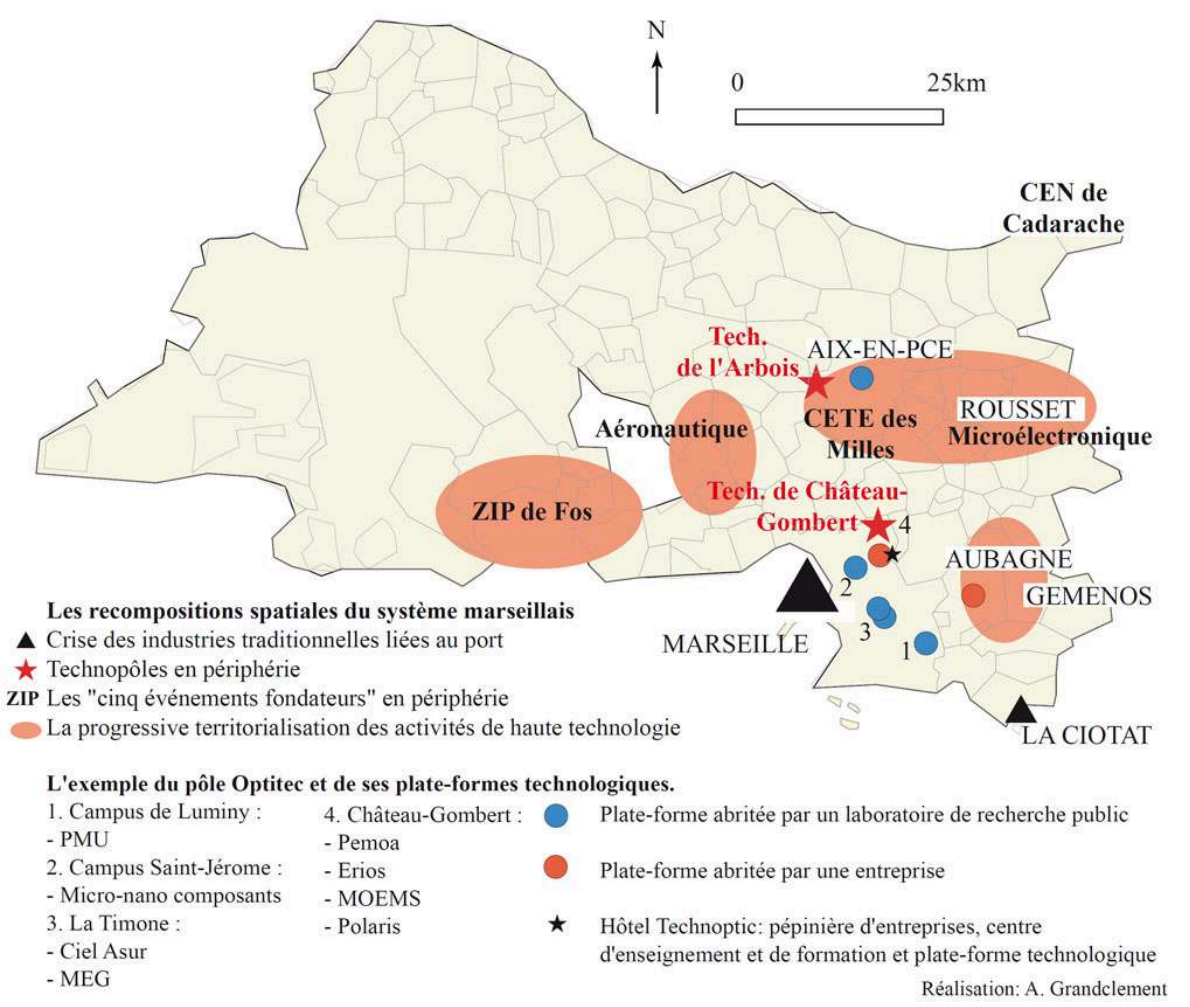

Le processus d'ancrage et d'endogénéisation de ces industries et la constitution de milieux locaux actifs parcourus de relations étroites entre les acteurs s'est en effet étalé sur une trentaine d'années, que ce soit dans la métropole marseillaise ou sur le technopôle de Sophia. Les crises cycliques de la microélectronique et la crise plus brutale des années 1990 à Sophia-Antipolis ont paradoxalement renforcé ce mouvement (Grondeau 2007 ; Ter Wal 2008) en accélérant les processus d'essaimage et en renforçant l'intervention des collectivités territoriales. L'attachement des cadres et ingénieurs à ces territoires a conduit nombre d'entre eux à créer leur propre entreprise, lors du départ des grandes entreprises étrangères, donnant naissance à une nébuleuse de petites entreprises et de sous-traitants, à l'image de Smart Packaging Systems ou d'Ion Beam Services, spin-offs de ST Microelectronics. Ce tissu de PME et de start-up innovantes, la circulation de la main d'œuvre, le rôle des grandes écoles régionales ont contribué à forger des réseaux d'interconnaissances qui ont accéléré la mise en œuvre de formes d'organisation collectives. 


\section{L'inégale organisation collective des secteurs et des territoires}

13 La labellisation des pôles de compétitivité ne se limite pas à l'identification d'un potentiel industriel, scientifique et universitaire mais reconnaît aussi l'existence d'une dynamique partenariale susceptible de le porter. La description de l'inégale structuration des réseaux collectifs d'un secteur et d'un territoire à l'autre permet d'inscrire la création des pôles dans la trajectoire des tissus productifs et relationnels et éclaire les choix des acteurs.

Deux processus peuvent être distingués dans la description de ces trajectoires, la constitution de milieux cohérents d'une part, l'institutionnalisation de formes d'action collective d'autre part. Le paragraphe précédent a déjà évoqué l'émergence de milieux qui accroissent l'ancrage territorial des activités au fur et à mesure des recompositions industrielles. Le renforcement des réseaux d'interconnaissance et la construction de normes et de valeurs partagées qui favorisent les relations de confiance (Grossetti et Bes 2002) sont un préalable indispensable aux initiatives collectives plus institutionnalisées qui peuvent ensuite alimenter un processus d'auto-renforcement.

De fait, les modalités de transformation des systèmes productifs décrites plus haut conditionnent les dynamiques d'organisation collectives. De manière paradoxale, l'ancienneté des tissus industriels et la proximité sociale peuvent même constituer un obstacle que ne connaissent pas les tissus d'acteurs plus récents. L'industrie des arômes et des parfums de Grasse est ainsi profondément ancrée dans le territoire et les entreprises demeurent pour une partie d'entre elles des entreprises familiales. Pourtant la culture locale du secret propre à cette industrie a limité l'apparition de formes d'organisation collective (Mendez et Bardet 2009). Dans le cas des anciens chantiers navals des Bouches du Rhône et du Var, la culture industrielle locale et l'ancrage social et territorial fort ont au contraire favorisé l'apparition d'un tissu de petites entreprises dans le secteur de la grande plaisance et de liens de coopération sous la forme du réseau Riviera Yachting Network (Garnier et Mercier 2008). La crise de la construction navale a renforcé la proximité entre les acteurs.

À ces processus spontanés et plus ou moins rapides s'ajoutent des initiatives et des projets volontaristes, souvent portés par des acteurs publics. Le cas de la microélectronique autour de Rousset-Gémenos illustre le développement simultané d'un milieu local et le rôle d'actions plus volontaristes (Daviet 2003). L'implication de l'État et le souci des collectivités territoriales de soutenir les hautes technologies et le bassin de Gardanne face à une série de crises cycliques ont rencontré la volonté de cadres des entreprises locales de renforcer l'ancrage territorial et la compétitivité de la filière. Cette dynamique collective d'organisation de la filière et d'approfondissement des relations s'est incarnée dans une série d'institutions et d'équipements structurants. L'association Arcsis, qui rassemble aujourd'hui les principaux acteurs de la filière, est l'héritière du Centre Régional d'Étude de Micro-électronique et Systèmes Interactifs (CREMSI), fondé en 1993 à l'initiative du Conseil Régional, et coiffe le Centre Intégré de Microélectronique de la région PACA (CIM PACA). Ce dernier abrite trois plates-formes technologiques mutualisées qui mettent des équipements de pointe à la disposition des acteurs scientifiques et industriels. Le soutien politique et financier de l'État et des collectivités a joué un rôle important dans la construction d'un milieu local, même si les efforts d'organisation collective des acteurs industriels et scientifiques ont été centraux. 
On observe donc un degré de structuration collective très variable d'un secteur et d'un territoire à l'autre. Certaines filières ont appris à mener des projets de recherche partenariale dès avant la création des pôles tandis que d'autres ne parvenaient pas à approfondir et faire évoluer des liens pourtant anciens. À la création des pôles, cette diversité recoupait également souvent des frontières sectorielles et/ou géographiques plus ou moins étanches. Dans le domaine de la construction navale, la moyenne plaisance n'entretenait quasiment aucune relation avec les grandes entreprises du secteur et les relations entre Toulon et La Ciotat étaient limitées. De la même manière, l'action d'Arcsis était concentrée sur les Bouches du Rhône et les relations de coopération entre Rousset et Sophia-Antipolis restaient à développer.

\section{La création des pôles de compétitivité}

\section{A. Des logiques de création en rupture avec les cadres sectoriels}

18 L'appel à projet national des pôles de compétitivité a connu en Provence-Alpes-Côte d'Azur le même succès qu'à l'échelle nationale, conduisant à la labellisation de 9 pôles. Au-delà du nombre, c'est le positionnement et les choix stratégiques opérés par les projets labellisés qui retiennent ici l'attention. Une première approche sectorielle classique permettrait de retrouver les grands secteurs de compétences de la région. Les projets présentés portent toutefois des innovations qui marquent des inflexions réelles dans la façon de concevoir et d'organiser le système productif régional. Ce processus résulte de la rencontre de la politique nationale, des mutations récentes des industries régionales et de la stratégie des collectivités territoriales qui ont guidé la définition du périmètre industriel et géographique des pôles. Elles ont également été élaborées en réponse au cahier des charges qui accompagnait l'appel à projet, en cherchant à satisfaire aux critères d'évaluation annoncés.

19 L'élément central de l'appel à projet national consistait en une injonction à former des pôles ayant une masse critique certaine (aussi floue que soit cette formule qui n'est jamais explicitée) et par conséquent à changer d'échelle par rapport aux formes d'organisation collectives existantes. Dans la mesure où cette politique avait ouvertement été décrite comme destinée à rassembler tout au plus dix à vingt pôles, cette exigence constituait un réel facteur discriminant aux yeux des acteurs locaux et une difficulté à surmonter pour la grande majorité des dossiers. Le processus de changement d'échelle et d'acquisition d'une taille suffisante a donc orienté en grande partie les projets de pôles de compétitivité. L'enjeu était de rassembler suffisamment d'acteurs et de compétences pour se hisser au niveau requis, ce qui faisait de la définition du périmètre industriel et géographique des futurs pôles une étape importante.

Les neuf pôles ne recoupent de fait pas strictement les limites sectorielles classiques (tableau 1). La première caractéristique partagée par les projets repose sur la volonté d'intégrer l'ensemble des chaînes de valeur depuis l'amont et la recherche fondamentale, jusqu'à l'aval. Le pôle agroalimentaire PEIFL se positionne ainsi « de la fourche à la fourchette ", le pôle Capénergies " de la production à la consommation d'énergie ». On peut alors identifier trois logiques dominantes. 
Tableau 1 : Les 9 pôles de compétitivité de la région PACA

\begin{tabular}{|c|c|}
\hline Pôle & Secteurs et filières concernés \\
\hline Capénergies & $\begin{array}{l}\text { Nucléaire, biocarburants, hydrogène, hydraulique, } \\
\text { éolien, bâtiment, habitat, solaire, géothermie }\end{array}$ \\
\hline Eurobiomed & $\begin{array}{l}\text { Toute la chaîne de valeur des sciences et technologies du vivant ; } \\
\text { industrie pharmaceutique, biotechnologies, équipement médical }\end{array}$ \\
\hline Optite & $\begin{array}{l}\text { Espace et aéronautique, énergie (nucléaire), mer, santé, télécoms } \\
\text { microélectronique, procédés industriels, environnement }\end{array}$ \\
\hline $\begin{array}{l}\text { Parfums Arômes } \\
\text { Senteurs Saveurs }\end{array}$ & $\begin{array}{l}\text { Production de plantes aromatiques, chimie fine, biotechnologies, } \\
\text { parfumerie, cosmétiques, agro- alimentaire }\end{array}$ \\
\hline Pégase & $\begin{array}{l}\text { Navigation des aéronefs, imagerie, motorisation, composants et } \\
\text { matériaux, énergies }\end{array}$ \\
\hline $\begin{array}{l}\text { Pôle Européen } \\
\text { d'Innovation Fruits et } \\
\text { Légumes }\end{array}$ & Agriculture, agroalimentaire, emballages, logistique, grande distribution \\
\hline Pôle Mer & $\begin{array}{l}\text { TIC, robotique et électronique embarquée, technologies sous-marines } \\
\text { optique-photonique, acoustique, énergies, biotechnologies, matériaux } \\
\text { et maintenance navale }\end{array}$ \\
\hline Pôle Risques & $\begin{array}{l}\text { A la convergence de secteurs variés allant de l'environnement aux } \\
\text { télécommunications, en passant par l'optique ou les technologies de la } \\
\text { mer }\end{array}$ \\
\hline $\begin{array}{l}\text { Solutions } \\
\text { communicantes } \\
\text { sécurisées }\end{array}$ & Microélectronique, télécommunications, logiciel et multimédia \\
\hline
\end{tabular}

21 Un premier ensemble de pôles s'est constitué selon une logique agrégative, par addition de composants préexistants. Ces pôles sont nés du rapprochement de plusieurs filières et/ou territoires. La géographie industrielle de la région conduit en effet souvent les deux à se recouper. Le pôle Parfums Arômes Senteurs Saveurs (PASS) fait ainsi figure sur le papier d'idéal-type de la transition vers les pôles de compétitivité. Il a été formé par le rapprochement de deux systèmes productifs locaux déjà constitués, l'un en Haute Provence autour de Manosque, le pôle Saveurs Senteurs, l'autre autour de Grasse, le pôle Arômes et Parfums. Ce mariage permettait également d'intégrer l'amont et l'aval de la filière. Le pôle Eurobiomed s'est de même organisé autour des deux clusters biomédicaux de Marseille et de Montpellier. Un second ensemble de pôles s'est organisé selon une logique plus strictement thématique, autour de secteurs émergents. Les pôles Mer et Risques correspondent à ce schéma. Il s'agissait dans ce cas de rassembler des acteurs issus de filières variées pour faire apparaître de nouvelles synergies sur la base des spécialisations régionales existantes (depuis la construction navale aux biotechnologies en passant par l'exploration sous-marine dans le cas du pôle Mer). Ce choix de stratégies innovantes conduit toutefois ces pôles à s'appuyer sur des réseaux beaucoup plus embryonnaires et sur des coopérations moins intenses et moins approfondies. Les autres pôles de la région ont adopté des logiques sectorielles plus traditionnelles même s'ils ont répondu à la nécessité de changer d'échelle. Le pôle Optitec est directement issu de l'association Popsud dans le domaine de l'optique et de la photonique. Le pôle Pégase s'inscrit lui aussi dans une logique sectorielle en rassemblant les acteurs de l'aéronautique autour d'Eurocopter et de Thales Alénia Space. 

de région d'une part, le Conseil Régional d'autre part. Le préfet était le dépositaire des grandes orientations de la politique nationale mais il est aussi intervenu grâce à sa connaissance du contexte et des enjeux locaux. Dans plusieurs cas, des divergences ont pu apparaître entre les projets portés par les acteurs industriels et la vision des acteurs publics. Dans le cas du pôle PASS, les syndicats professionnels, puissants dans ces industries, ont joué un rôle majeur dans la construction du dossier et sont à l'origine du rapprochement de l'amont de la filière en Haute Provence et des industriels des arômes et des parfums de Grasse. Le préfet de région et le préfet de département défendaient quant à eux la vision d'un pôle entre Grasse et Sophia-Antipolis, davantage orienté vers les sciences de la vie et qui aurait intégré des capacités de Recherche et Développement (R\&D) bien plus importantes, contrairement au projet de pôle autonome voulu par les syndicats professionnels. Si cette approche n'a pas été retenue, la préfecture est en revanche intervenue pour intégrer dans le projet le Club des Entrepreneurs de Grasse, la structure d'animation du Système Productif Local (SPL) Arômes et Parfums de Grasse. Son absence initiale était liée à la césure qui existait localement entre les grandes entreprises de Grasse représentées par le syndicat PRODAROM (Syndicat National des Fabricants de Produits Aromatiques) et le SPL qui rassemblait plutôt les petites et moyennes entreprises de la filière. Le schéma théorique d'un rapprochement de deux SPL pour donner naissance au pôle cache donc des jeux d'acteurs et des équilibres politiques complexes. Dès 2004, un rapport au conseil régional sur les modes de transition des systèmes productifs de la région estimait que " Grasse [avait] très clairement raté l'arrimage sur Sophia-Antipolis » (Garnier et al. 2004, p.290). Dans le cadre d'une politique qui met en avant l'innovation et les capacités de R\&D, ces choix se révèlent lourds de conséquences. Les modes de fonctionnement hérités et les représentations des acteurs industriels peuvent clairement faire obstacle à la mise en œuvre de forme d'organisations innovantes, malgré l'intervention des collectivités et de l'État. Là encore, les trajectoires industrielles et territoriales jouent un rôle important, même si le poids du politique ne doit pas être négligé.

\section{L'exemple du pôle SCS}

25 Seul pôle mondial de la région PACA, le pôle Solutions Communicantes Sécurisées (SCS) illustre bien l'importance de ces logiques plurielles. Ce sont en effet quatre filières qui se sont réunies, la microélectronique, les télécommunications, les logiciels et le multimédia. La mise en œuvre de la stratégie résumée par la formule « du silicium aux

Revue Géographique de l'Est, vol. 54 / 1-2 | 2014 
usages " se traduit donc par une rupture nette avec les logiques sectorielles traditionnelles. Le rapprochement dans ce cas est aussi géographique puisqu'il met en relation au sein du pôle les Bouches-du-Rhône autour du cluster de microélectronique de Rousset-Gémenos et les Alpes-Maritimes autour du technopôle de Sophia-Antipolis. Le pôle est ainsi le produit des opérations d'aménagement du territoire volontariste menées par l'État dans les années 1970 et 1980 et des dynamiques de constitution de milieux industriels et scientifiques cohérents, organisés autour de formes d'organisation collective développées. Les associations professionnelles issues de l'ancrage progressif de ces greffes industrielles révèlent la persistance des logiques sectorielles et logiques géographiques comme le montre la figure 2. Elles ont toutefois joué un rôle majeur lors de la création du pôle en facilitant la mise en relation à l'échelle régionale de filières et de territoires encore peu habitués à travailler ensemble. Si le Conseil Régional a défendu l'intégration du secteur du logiciel et du multimédia dans le pôle et des associations professionnelles Medmultimed, Medinsoft et Baby Smart dans son conseil d'administration, le rapprochement des deux pôles de l'ouest et de l'est de la région et des acteurs de la microélectronique et des télécommunications n'a pas posé de problème. L'État et la Région ont fortement poussé dans ce sens mais les acteurs industriels n'y étaient pas réticents. Ces derniers étaient des hommes nouveaux, peu sensibles aux clivages culturels et socio-économiques anciens (Daviet, 2005a), et dont les préoccupations se focalisaient sur la recherche de solutions permettant de contrer les crises sectorielles (Daviet 2005b ; Daviet et Monge 2010). On note ainsi au passage que les pôles entérinent le rôle croissant des services à forte intensité de connaissance (SFIC) au sein desquelles l'INSEE rassemble les activités de services technologiques et de services intellectuels, dont les secteurs du multimédia et du logiciel sont un bon exemple.

Figure 2 : Des réseaux d'acteurs à l'origine du pôle SCS

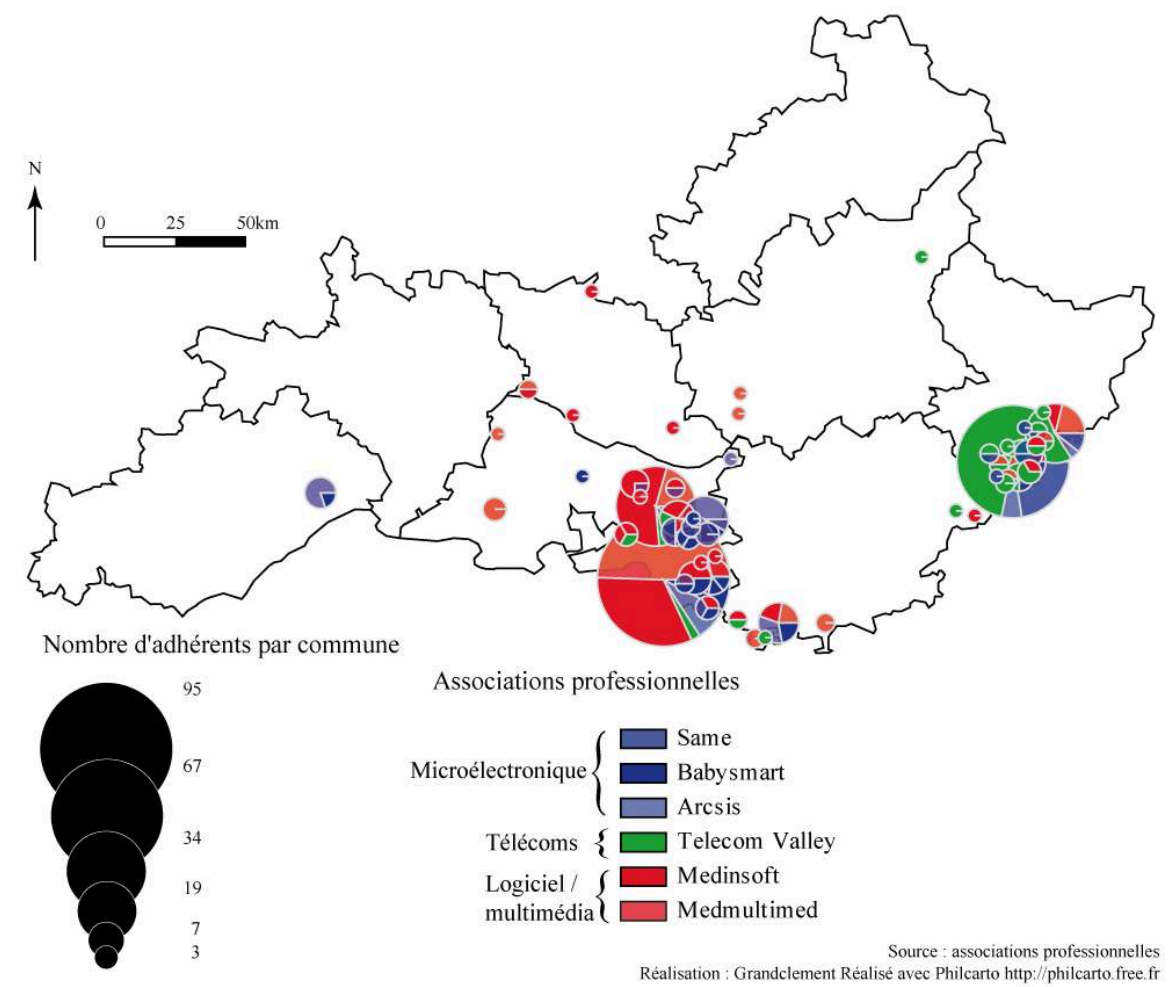


La cartographie des collaborations au sein du pôle, réalisée à partir des projets de recherche associant entreprises et laboratoires, montre à la fois la prégnance de la géographie industrielle régionale et l'inflexion introduite par la politique des pôles (figure 3). On distingue en effet nettement la bipolarisation régionale entre Nice-Sophia et Aix-Marseille mais aussi le développement de liens entre ces territoires et l'ouverture accélérée des réseaux à de nouveaux territoires, jusque dans l'Hérault.

Figure3 : Les réseaux d'innovation du pôle SCS

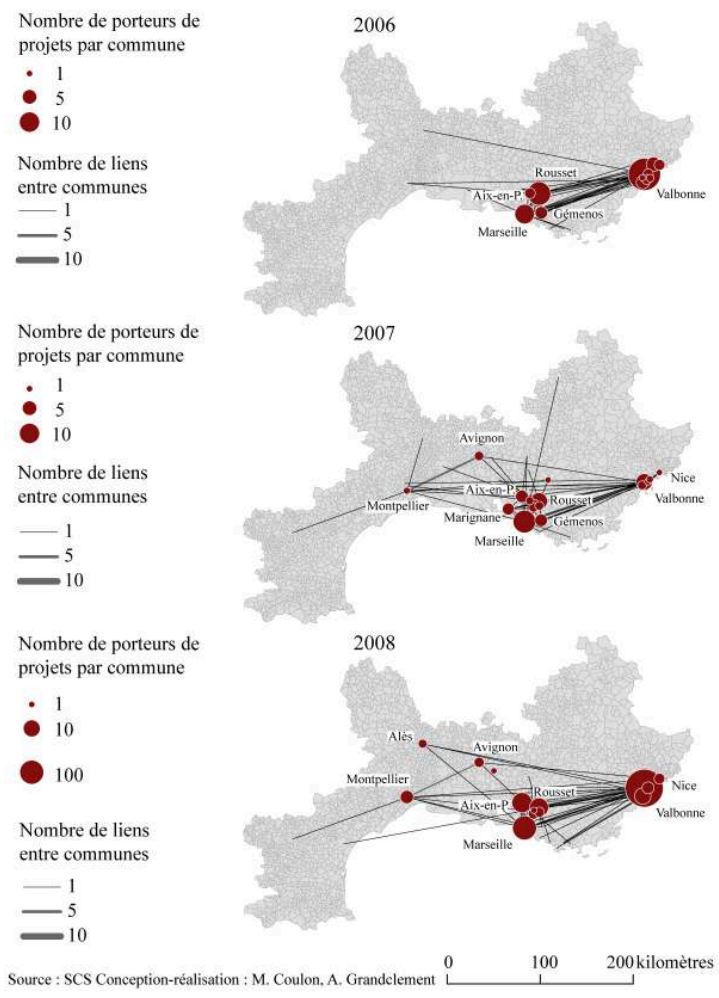

27 Le rapprochement des acteurs de la micro-électronique de Rousset et de ceux de Sophia-Antipolis n'a pourtant pas gommé tous les clivages qui existaient entre ces deux territoires. Le pôle possède ainsi une antenne à Sophia-Antipolis et une autre à Rousset et son siège se déplace tous les deux ans entre les deux territoires. De la même manière, la présidence est occupée en alternance et pour une période de deux ans par un représentant de Rousset et par un représentant de Sophia-Antipolis. Ces équilibres se sont révélés très fragiles lors des premières années de fonctionnement du pôle : en 2007, une deuxième élection du président a dû être organisée après que les acteurs des Bouches-du-Rhône se furent abstenus, obligeant le préfet à invalider le résultat au vu du faible taux de participation. L'élection d'un représentant de Sophia ne semblait donc pas aller de soi. Les équilibres politiques et territoriaux restent donc un enjeu important, même si ces tensions initiales se sont rapidement effacées. Elles n'ont d'ailleurs pas empêché la mise en place de solutions pragmatiques dans le fonctionnement quotidien du pôle. Une partie des réunions qui rassemblent des acteurs de l'Est et de l'Ouest de la région se tient à Brignolles, à mi-chemin entre les deux villes pour diminuer les déplacements. Cette pratique constitue un exemple marquant de proximité temporaire ainsi que l'a définie André Torre (Torre 2008). 


\section{Les pôles, acteurs de l'organisation spatiale des systèmes productifs}

\section{A. La diversité des stratégies des pôles} enjeux industriels et productifs de la filière aéronautique et du territoire. Le poids du grand donneur d'ordres qu'est Eurocopter et ses besoins industriels mais aussi la présence d'un tissu de petites et moyennes entreprises (PME) et de start-up ont entre autre conduit Pégase à mettre en place deux initiatives originales pour structurer et organiser la filière aéronautique et renforcer les atouts du territoire. Le programme Strat'aéro a pour but de rendre plus efficace la chaîne de sous-traitance régionale en en identifiant les critères retenus par Eurocopter dans le choix de ses sous-traitants et en accompagnant les PME dans les mutations et les repositionnements nécessaires pour satisfaire ces exigences ou gagner de nouveaux contrats. Le projet «Université des Petits Maîtres d'œuvre " accompagne quant à lui les jeunes entreprises depuis l'incubation pour leur faire atteindre une taille critique et les faires bénéficier de l'expérience d'entreprises matures. Dans d'autres pôles, c'est la formation qui est au cœur des préoccupations. Le pôle Mer est à l'origine de l'ouverture d'un MBA (maîtrise en administration des affaires) Maritime au sein de l'école de commerce de Toulon et d'une formation de chaudronnier. Le pôle PASS, quant à lui, a créé une commission formation qui doit réfléchir aux enjeux des évolutions de la filière tels que l'apparition de nouveaux métiers ou la recherche de nouvelles compétences.

Les pôles portent par ailleurs une même politique de multiplication des plates-formes technologiques et d'équipements mutualisés. Ce mouvement répond en partie aux besoins des industriels et des laboratoires de recherche qui ne sont plus en mesure de financer seuls ce type d'équipements mais il a aussi vocation à stimuler la mise en réseau des acteurs et l'établissement de coopérations suivies. Le pôle Optitec parle d'un «maillage de plates-formes technologiques sur le territoire régional » que constituent les plates-formes PACA2M (à Aubagne dans les locaux de l'entreprise Cilas), Optilab (au sein de l'École Nationale Supérieure d'Arts et Métiers d'Aix-en-Provence), Ciel-Asur dans les locaux du laboratoire Lasers - Plasma et Procédés photoniques puis sur le campus de la Timone), Polaris (dans les locaux du Laboratoire d'Astrophysique de 
Marseille), PEMOA ${ }^{5}$ (à Château-Gombert). Le pôle est aussi à l'origine de la création à Marseille de " l'Hôtel Technoptic " qui associe un incubateur d'entreprises et deux plates-formes technologiques mutualisées destinées à accélérer la croissance de la filière photonique (figure 1). Là encore, on peut lire dans l'action du pôle Optitec l'importance des spécificités de la jeune filière de l'optique-photonique en PACA qui travaille à se doter des formes collectives et des outils que d'autres filières plus anciennes ont déjà su construire. Au-delà de la recherche, c'est en outre de la question de la production que s'emparent les pôles.

\section{B. D'une géographie de l'innovation à une géographie de la production}

Dans le contexte des débats sur la désindustrialisation, et à l'initiative du nouveau ministère du redressement productif, la troisième phase de la politique des pôles de compétitivité lancée en 2013 met fortement l'accent sur la création d'emplois et le passage de la recherche à la production industrielle. S'il est trop tôt pour savoir si le passage espéré d' " usines à projets » à des « usines à produits » se concrétisera, on peut constater que cet enjeu est une préoccupation ancienne des pôles.

Les pôles sont partie prenante d'un nombre croissant de projets de démonstrateurs grandeur nature aux côtés des collectivités territoriales et des entreprises de la région. Ces projets peuvent être liés à la concrétisation d'innovations technologiques issues de projets de recherche mais sont pour une partie des opérations d'aménagement ou de développement d'équipements. Parmi eux, on peut citer la participation de Capénergies au projet de Vallée des Energies Nouvelles en Haute-Provence ou l'implantation d'une plate-forme expérimentale pour promouvoir les énergies renouvelables, optimiser la production locale des ressources en énergie et leur stockage, et piloter l'offre et la demande en électricité. Cette dernière opération (projet Premio) réunit Capénergies, une communauté de communes et une douzaine de communes parmi lesquelles Lambesc, Gardanne et Fréjus. La construction de tels démonstrateurs est considérée comme un atout pour conquérir des marchés puisqu'ils permettent de démontrer à d'éventuels clients l'efficacité des innovations portées par les membres des pôles.

La volonté des pôles de compétitivité de développer les atouts et l'attractivité de leur territoire les conduit à en outre essayer d'organiser ce territoire, au-delà du seul champ de la R\&D. L'ambition du pôle Pégase d'« élaborer un écosystème compétitif maillant le réseau de grandes entreprises, de centres de recherche et d'essais et le tissu de PME innovantes existantes ou à créer dont dispose la région PACA » en est un bon exemple. Cette stratégie s'appuie, outre les plates-formes technologiques déjà évoquées, sur la création de zones d'activités destinées à accueillir de nouvelles entreprises. Le pôle Pégase porte donc la création d'une zone d'activités dédiée à la sous-traitance à Marignane et d'un campus de formation autour du maintien des aéronefs moyens en condition opérationnelle. Son principal projet, baptisé technopôle du pôle Pégase, associe en périphérie de l'aéroport d'Avignon une zone d'activités de 17 ha dédiée à l'aéronautique et une pépinière d'entreprises qui accueillera de jeunes pousses du secteur. Le pôle prévoit d'accompagner les entreprises en proposant une exonération des redevances domaniales réservée aux membres du pôle. On retrouve le souci de ce pôle d'organiser la filière, y compris en prenant en charge les aspects fonciers pourtant éloignés de sa mission première. 

géographie des pôles est indissociable d'une géographie des activités productives. Les technopôles de la région (Château-Gombert pour Marseille, le technopôle de l'Arbois pour Aix et Sophia-Antipolis) sont des pôles structurants et marquent l'inscription des pôles dans des trajectoires anciennes de localisation des entreprises et d'organisation des filières. Il est ainsi intéressant de constater que les pôles qui ne disposent pas de cette antériorité et de la légitimité qu'elle confère ont fait de ce type de territoires le cœur de leur stratégie. Le pôle PEIFL qui ne peut pas s'appuyer sur des réseaux de collaboration anciens met en avant le technopôle Agroparc d'Avignon comme pivot de son action. Dans une logique similaire, le pôle Mer a contribué à réactiver le projet toulonnais de Technopôle de la Mer qui faisait figure de serpent de mer depuis une dizaine d'années. Il est particulièrement marquant de constater que les pôles, malgré la rupture affiché par la politique nationale, font une place à ces formes territoriales anciennes, alors qu'on s'interrogeait sur leur obsolescence (Fache, 2006).

\section{Conclusion}

Loin de n'être que des réseaux d'innovation, les pôles de compétitivité s'inscrivent dans la trajectoire industrielle et territoriale des systèmes productifs régionaux. Les mutations récentes de ces derniers conditionnent en grande partie le fonctionnement des pôles qui contribuent en retour à transformer l'organisation spatiale des activités productives. Si les projets de recherche collaborative sont au cœur de l'organisation spatiale des pôles (Grandclement, 2012), il serait erroné de ne pas articuler cette géographie des réseaux d'innovation à une géographie des activités productives qui révèle l'acuité de questionnements traditionnels de la géographie industrielle et de l'aménagement du territoire. L'aménagement de zones d'activités, d'incubateurs, la construction d'équipements structurants, l'animation, voire la création de technopôles et la mise en réseau de ces lieux pour mailler le territoire ne sont pas rendus obsolètes par l'avènement d'hypothétiques réseaux hors-sol mais demeurent inséparables des politiques d'innovation. Penser conjointement la géographie de l'innovation et la géographie des activités productives constitue ainsi un des défis majeurs de la recherche autant que des politiques.

\section{BIBLIOGRAPHY}

Albertini J.B., 2006, « De la DATAR à la nouvelle DIACT : la place des questions économiques dans la politique d'aménagement du territoire ", Revue française d'administration publique, n¹19, p. 415-426.

Blanc C., 2004, Pour un écosystème de la croissance, Paris, La Documentation Française, 81 p.

Boquet R., Mendez A., Mothe C., Bardet M., 2009, « Pôles de compétitivité constitués de PME : quelle gouvernance pour quelle performance?», Management et Avenir, n5, p.227-244.

Revue Géographique de l'Est, vol. 54 / 1-2 | 2014 
Carroué L., 2014, La France. Les mutations des systèmes productifs, Paris, Armand Colin, 235 p.

Cohen E., 2007, « Industrial policies in France, the old and the new », Journal of Industry Competition and Trade, Vol.7, $\mathrm{n}^{\circ} 3$, p. 213-227.

Daumalin X., Girard N., Raveux O. (dir), 2003, Du savon à la puce : l'industrie marseillaise du XVIIe siècle à nos jours, Marseille, J. Lafitte, $379 \mathrm{p}$.

Daviet S., 2003, Mise en cohérence, fragilités et nouvelles dynamiques de la microélectronique en région PACA, Étude pour la MDER, Université de Provence, 96 p.

Daviet S., 2005a, « Gestion des espaces et trajectoire entrepreneuriale : le cas des managers de la microélectronique ", in Aubert P., Chastagnaret G., Raveux O. (dir), Construire des Mondes. Élites et espaces en Méditerranée, Aix en Provence, Publications de l'Université de Provence, p.309-324.

Daviet S., 2005b, Industrie, culture, territoire, Paris, L'Harmattan, 208 p.

Daviet S., Monge R., 2010, « From “evolutionary turn" to "territorial resources”: the new trajectories of innovation in Provence, France ", Geography Compass, Vol. 4, n¹0, p.1497-1512.

Fache J., 2002, Les territoires de la haute technologie : éléments de géographie, Rennes, Presses Universitaires de Rennes, $157 \mathrm{p}$.

Fache J., 2006, « Technopôles : un concept dépassé ?», Norois, n²00, p.7-9.

Fen-Chong S., Weil T., 2008, « Comment appréhender la diversité des pôles ? », Séminaire du 17 janvier, Observatoire des Pôles de Compétitivité, $12 \mathrm{p}$.

Garnier J., 2005, « Proximités lourdes, proximités légères : une trajectoire de l'appareil productif dans l'aire métropolitaine marseillaise », Géographie Économie Société, Vol.7, n4, p.365-380.

Garnier J., 2011, Un appareil productif en mutation : les 50 ans qui ont tout changé en Provence-AlpesCôte-d'Azur, Paris, Économica, 229 p.

Garnier J., Mercier D., 2008, « La création d'entreprises entre autonomisation et intégration : comparaison de deux processus dans l'aire métropolitaine marseillaise », Géographie Économie Société, Vol.10, n¹, p.87-102.

Garnier J., Zimmermann J.B., 2006, «L'aire métropolitaine marseillaise et les territoires de l'industrie », Géographie Économie Société, Vol.8, n², p.215-238.

Garnier J., Lamanthe A., Lanciano-Morandat C., Mendez A., Mercier D., Rychen F., 2004, Les modes de transition du tissu productif régional en Provence-Alpes-Côte d'Azur. Une étude comparative de quatre sites, Rapport de recherche pourle Conseil Régional de Provence-Alpes-Côte d'Azur, LEST, 394 p.

Grandclement A., 2012, Géographie des pôles de compétitivité : réseaux et territoires de l'innovation, Thèse de doctorat de géographie, université d'Aix-Marseille, $625 \mathrm{p}$.

Grondeau A., 2007, Contribution à une géographie critique des territoires de haute technologie, Thèse de doctorat de géographie, université Paris 10, $402 \mathrm{p}$.

Grossetti M., Bes M.P., 2002, « Proximité spatiale et relation science-industrie : savoir tacite ou encastrement? ", Revue d'Économie Régionale et Urbaine, n5, p.777-788.

Guillaume R., 2008, « Des systèmes productifs locaux aux pôles de compétitivité : approches conceptuelles et figures territoriales du développement ", Géographie Économie Société, Vol.10, n³, p.295-309.

Laffitte P., 2003, « Technopoles et clusters. Pour la croissance par l'innovation », Réalités Industrielles, $\mathrm{n}^{\circ}$ de décembre, p.39-41. 
Mendez A., Bardet M., 2009, «Quelle gouvernance pour les pôles de compétitivité constitués de PME », Revue française de gestion, $\mathrm{n}^{\circ} 190, \mathrm{p} .123-142$.

Morel, B., 1999, Marseille, naissance d'une métropole, Paris, L'Harmattan, 221 p.

Pecqueur B., 2008, «Pôles de compétitivité et spécificité de la ressource technologique : une illustration grenobloise ", Géographie Économie Société, Vol.10, n³, p.311-326.

Plunket A., Torre A., 2009, « Les pôles de compétitivité ou le retour ambigu des déclinaisons locales de la politique industrielle française », Economia e Politica Industriale, n³, p.159-177.

Rychen F., Zimmermann J.B., 2001, « Une approche territoriale du développement industriel : la microélectronique dans l'aire métropolitaine marseillaise ", Revue d'Économie Régionale et Urbaine, $\mathrm{n}^{\circ} 1, \mathrm{p} .115-134$.

Ter Wal A., 2008, «Cluster emergence and network evolution: a longitudinal analysis of inventor network in Sophia-Antipolis », Papers in Evolutionary Economic Geography, Utrecht University, $25 \mathrm{p}$.

Torre A., 2008, « On the role played by temporary geographical proximity in knowledge transfer ", Regional Studies, Vol.42, n6, p.869-889.

\section{NOTES}

1. Les chantiers de La Ciotat et La Seyne-sur-Mer ferment en 1989 après une dizaine d'années de crise profonde, marquée notamment par la disparition de la Normed. L'exploitation minière cesse définitivement à Gardanne en 2003

2. Le processus de spin-off ou essaimage consiste en la création d'une nouvelle entreprise par scission avec une entreprise plus grande, souvent avec le soutien de celle-ci. Dans le champ des hautes technologies, il consiste souvent à développer une activité ou appliquer une innovation que l'entreprise mère n'a pas retenue

3. On peut toutefois signaler le rôle de l'installation du Centre d'Études Nucléaires (CEN) du Commissariat à l'Énergie Atomique (CEA) à Cadarache et du Centre d'Études Techniques de l'Équipement sur le site des Milles

4. à laquelle on peut substituer l'implantation de la microélectronique à Rousset pour rester dans le champ industriel

5. Plate-forme D'Equipements Mutualisés en Optique Adaptative

\section{ABSTRACTS}

This paper focuses on the French competitiveness cluster policy. It questions the tension between the spatial anchorage of firms and their implication in science-industry networks of "competitiveness clusters". It shows that the recent spatial and industrial transformations of the regional production system are a major key to understanding the way "competitiveness clusters" were created and work. Far from being only innovation networks, "competitiveness clusters" are a major actor of the spatial organization of the production system. 
Cet article interroge la tension sensible dans la politique des pôles de compétitivité entre logiques territoriales d'ancrage des entreprises et logiques réticulaires d'insertion dans des réseaux de recherche science-industrie. En s'appuyant sur l'exemple de la région ProvenceAlpes-Côte d'Azur (PACA), il montre que les mutations spatiales et industrielles récentes des systèmes productifs régionaux sont une clé de lecture essentielle de la construction et du fonctionnement des pôles de compétitivité. Loin de n'être que des réseaux d'innovation, les pôles de compétitivité sont aussi un des acteurs de l'organisation des systèmes productifs.

\section{INDEX}

Mots-clés: pôles de compétitivité, Provence-Alpes-Côte-d'Azur, réseaux d'innovation, systèmes productifs

Keywords: competitiveness clusters, innovation networks, production system, Provence-AlpesCôte-d'Azur

\section{AUTHOR}

\section{ANTOINE GRANDCLÉMENT}

UMR 7303 TELEMME - ENS de Lyon - 15 Parvis René Descartes BP 700069342 Lyon Cedex 07 antoine.grandclement@ens-lyon.fr 